OPEN ACCESS

Edited by:

Fang Pan,

Shandong University, China

Reviewed by:

Tao Liu,

Hainan General Hospital, China

Wei Li,

Shanghai Jiao Tong University, China

*Correspondence:

Yuzhen Xu

tianyayizhe@126.com

${ }^{+}$These authors have contributed equally to this work

Specialty section:

This article was submitted to

Neuropharmacology,

a section of the journal

Frontiers in Pharmacology

Received: 30 November 2021 Accepted: 24 December 2021

Published: 15 February 2022

Citation:

Tao P, Ji J, Gu S, Wang Q and Xu Y (2022) Progress in the Mechanism of

Autophagy and Traditional Chinese Medicine Herb Involved in Dementia.

Front. Pharmacol. 12:825330.

doi: 10.3389/fphar.2021.825330

\section{Progress in the Mechanism of Autophagy and Traditional Chinese Medicine Herb Involved in Dementia}

\author{
Pengyu $\mathrm{Tao}^{1 \dagger}$, Jing $\mathrm{Ji}^{2 \dagger}$, Simeng $\mathrm{Gu}^{3 \dagger}$, Qian Wang ${ }^{4}$ and Yuzhen $\mathrm{Xu}^{5 *}$ \\ ${ }^{1}$ Basic Medical School, Shanghai University of Traditional Chinese Medicine, Shanghai, China, ${ }^{2}$ Department of Nephrology, \\ Yueyang Hospital Affiliated to Shanghai University of Traditional Chinese Medicine, Shanghai, China, ${ }^{3}$ Department of Psychology, \\ Jiangsu University Medical School, Zhenjiang, China, ${ }^{4}$ Postdoctoral Workstation, Department of Central Laboratory, Taian City \\ Central Hospital, Shandong First Medical University and Shandong Academy of Medical Sciences, Taian, China, ${ }^{5}$ Department of \\ Rehabilitation, The Second Affiliated Hospital of Shandong First Medical University, Taian, China
}

Dementias is a kind of neurodegenerative disease, which occurs among the aging population. Current therapeutic outcome for dementia is limited. The medical use of herbal plant has a rich history in traditional Chinese medicine practice for thousands of years. Herbal medicine (HM) may provide a positive effect for prevention and treatment in dementia. As an alternative treatment to dementia, there has been a growing interest in $\mathrm{HM}$ extracts in scientific community as a result of its promising study results, mainly in animal experiment. At the molecular level, HM extracts trigger autophagy and reduce generation of reactive oxygen species (ROS) while inhibiting inflammation and reduce neurotoxicity. Experiments both in vivo and in vitro have identified certain potential of HM extracts and natural products as an important regulator factor in mediating autophagy, which might contribute to the improvement of dementia. This brief review not only summarizes the mechanism of autophagy in dementia but also offers a general understanding of the therapeutic mechanism of HM extracts in treating dementia and evaluates the potential clinical practice of $\mathrm{HM}$ in general.

Keywords: autophagy, Chinese herbal medicine extracts, dementia, inflammation, oxidative stress, apoptosis

\section{INTRODUCTION}

Dementias, mainly including Alzheimer's disease (AD) and vascular dementia $(\mathrm{VaD})$ (the two major form of dementia), are characterized by memory loss, damaged judgment, and language barrier seriously affecting daily life (Xu et al., 2019). It is estimated by the WHO report based on epidemiological data that the number of people across the world suffering from dementias will jump to 81.1 million by 2040 (Le Couteur et al., 2020). It is universally acknowledged that heart attack, stroke, atherosclerosis, hypertension, obesity, smoking, and cardiac problems are vital risk factors contributing to the cause of vascular dementia (Sahyouni et al., 2021). Clinically, dementia covers a wide range of brain abnormalities, ranging from slow progressive loss of memory, cognitive function, to a failure to conduct personal daily activities. The hallmark of dementia is characterized by accumulation of $\beta$-amyloid $(A \beta)$ and hyper-phosphorylated Tau protein. The overexpression of these hallmarks often indicate neurodegenerative disorders that may turn into severe pathology. Thus, targeting these hallmarks may relieve the symptoms of dementias on the whole, and triggering autophagy by HM extracts is likely to be an alternative treatment for dementias (Padilla, 2019; Paik et al., 2020). 
Autophagy has been interfered in the pathogenesis of dementias ( $\mathrm{He}$ and Klionsky, 2009). It is generally assumed that autophagy is activated and acts as a protective mechanism in response to stimulating factors, thus attenuating toxic damage to the neuron. A variety of methods can be the promoter of autophagy, such as less food intake, regular sport, rapamycin, and AMP-activated protein kinase (AMPK)-activated protein kinase (Papandreou et al., 2008; Wang et al., 2021). However, once the autophagy pathway is impaired by stroke, atherosclerosis, and hypertension, this might lead to the progressive accumulation of toxic proteins in neuron, which finally contribute to the development of $\mathrm{VaD}$ (Romeo et al., 2019). Moreover, the activation of autophagy plays a key role in protecting and maintaining vascular integrity, and inhibition of autophagy is acting as a negative regulator in vascular degeneration, aging, and related pathological conditions (Levine and Kroemer, 2008; Ju and Weihl, 2010; Wang et al., 2021).

The incidence rate and mortality of dementias experienced a fast growth year by year. Current therapies simply offer symptomatic relief and fail to achieve a desired efficacy against dementias. Thus, there is an urgent need to seek an alternative treatment (Levine and Kroemer, 2008). Traditional Chinese medicine (TCM) is rooted in yin-yang theory and has been broadly used for the prevention and treatment of neurodegenerative disease in China for centuries (Han et al., 2012; Wang et al., 2021). As some Chinese medicines act as alternative approach in treating dementia and show effects on relieving dementia-related syndrome (Bai and Zhang, 2021), it has drawn great attention among researchers. It is the plant elements or extracts that carry out therapeutic function ( $\mathrm{Li}$ and Zhang, 2009). The derivations of medicinal herbs have shown potential in alleviating dementia; the herb-plant-derived natural products tested in clinical trials have obtained positive results of improving dementias (Lin et al., 2017). Recently, studies have suggested that medicinal herb extracts could slow down the development of dementia via activating different pathways, which might provide a guideline of TCM for $\mathrm{VaD}$ therapy (Shen and Li, 2005; Zhu et al., 2006).

\section{THE ROLE OF AUTOPHAGY AND ITS RELATED SIGNALING PATHWAY IN DEMENTIA}

Autophagy is a highly conserved self-digestion process involved in physiology to maintain cellular homeostasis via delivering dispensable or potentially damaged intracellular components, such as proteins and organelles to lysosomes for degradation, clearance, and recycling ( $\mathrm{He}$ and Klionsky, 2009). Macroautophagy, microautophagy, and chaperone-mediated autophagy are three main forms of autophagy, all of which vary from their mechanisms to functions (Morita et al., 2010). Of these three types, macroautophagy is the most intensively studied autophagy process (Wang et al., 2020a); thus, it is generally referred to as autophagy. Autophagy is a normal physiological activity occurring in most cells to maintain homeostasis. But under some extreme situation, autophagy is usually activated to act as an adaptive and protective mechanism in alleviating damage of cells exposed to risky factors, such as oxidative stress, inflammation, or metabolic disorders (Morita et al., 2010). Researchers found that the dysfunction of autophagy contributes to the pathogenesis of neurodegenerative disease, such as vascular dementia (Figure 1). Thus, restoring autophagy activation may improve the prognosis of dementia (Cuervo, 2013; Caberlotto and Nguyen, 2014).

The mammalian neuron system depends on autophagy to maintain its normal functions and homeostasis (Jiang and Chang, 2019). Numerous specific-gene knockout animal models have been established to show that deletion of core autophagy-related genes leads to neonatal and embryonic lethality (Alirezaei et al., 2008). Three major steps are necessary for the formation of autophagosome: initiation, nucleation, and elongation. With more than 30 autophagy-related genes (ATGs) participating in autophagy activation, this complex process is strictly regulated to keep dynamic balance between synthesis and degradation, use, and recycling of intracellular substances (Caberlotto and Nguyen, 2014). The ULK1/2-ATG13-FIP200-ATG101 complex is important for activating autophagy. The Becl-1-PI3K complex is necessary for the phagophore nucleation step. The Atg12-Atg5-Atg16 complex and LC3/Atg8 cascade are employed in the autophagosomes elongation step (Cuervo, 2013; Liu et al., 2014; Nussenzweig et al., 2015). The conversion of LC3-I to LC3-II is recognized as a marker of autophagy activation and autophagosome formation (Levine and Kroemer, 2008).

mTOR is the best known mammalian target of rapamycin and also considered one of the most significant autophagy regulators in eukaryotic cells that includes two mTOR complexes, the mTOR complex 1 (mTORC1) and complex 2 (mTORC2); mTORC1 is the most extensive studied complex (Cao, 2010). Under most normal conditions, mTORC1 suppresses autophagy function via phosphorylating ULK1 and thereby inhibiting its activity (Alers et al., 2012). However, once cells are exposed to oxidative stress stimuli, the autophagy process can be initiated via the inactivation of mTORC1 (Kim et al., 2011). In the nervous system, the autophagy function can be neuroprotective via the inhibition of mTORC1. The high expression of mTORC1 is frequently observed in animals with nervous system diseases, such as vascular dementia (Guo et al., 2021; Perluigi et al., 2021). Thus, autophagy activation with the inhibition of mTOR expression can lead to neural tissue protection (Cao, 2010). Results from experimental models of $\mathrm{AD}$ indicated that disease progression seems to have a connection with dysfunctional autophagic processes and be improved by inhibition of mTOR activity (Joungmok and Kun-Liang, 2019).

Sirtuin1 (SIRT1), a homolog of Sir2, regulates energy metabolism, longevity, and autophagy in an NAD+-dependent manner (Ji et al., 2020). The sirt1 activity promotes life survival in organisms and protects neuronal cells from oxidative stress (Salminen and Kaarniranta, 2009). SIRT1 is responsible for the homeostasis of neural systems (Kim et al., 2007). If sirt1 activity is lost, this leads to impaired cognitive abilities, elevated $\mathrm{A} \beta$ production, and accumulation in $\mathrm{AD}$ patients (Qin et al., 2006). The increased sirtl level can induce autophagy via 


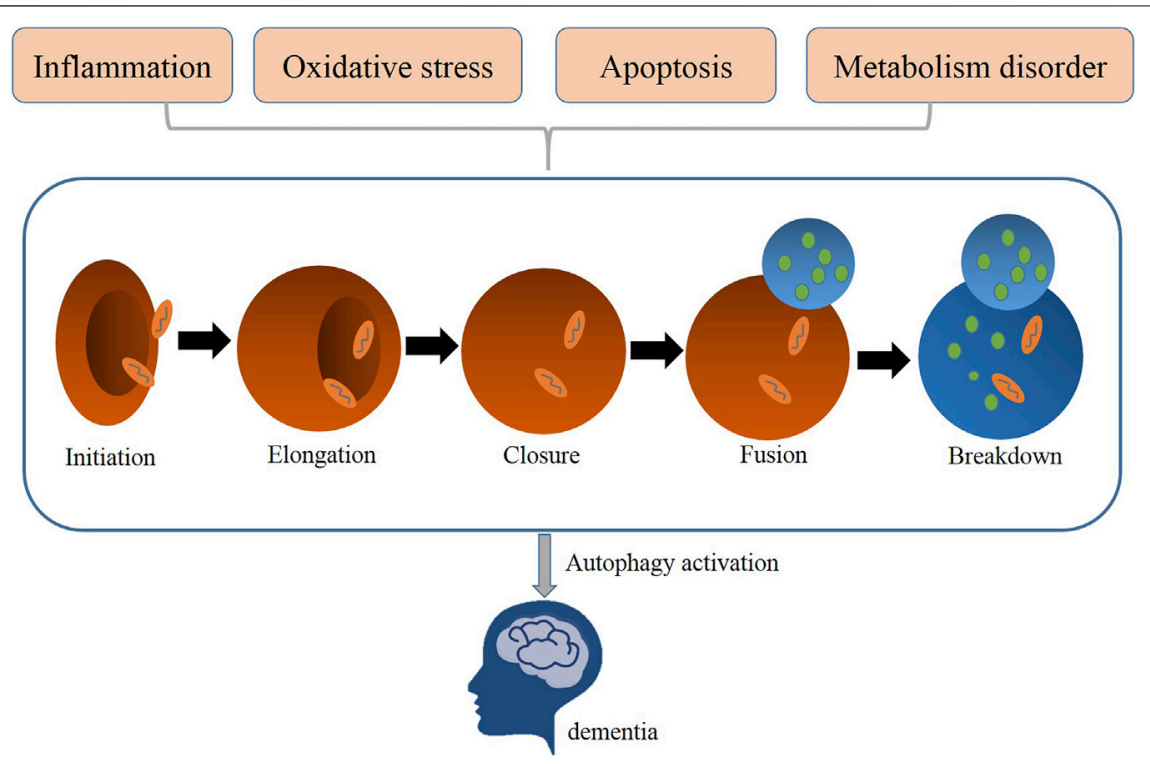

FIGURE 1 | Related pathological processes (metabolism disorder, oxidative stress, apoptosis, inflammation) mediated autophagy in dementia.

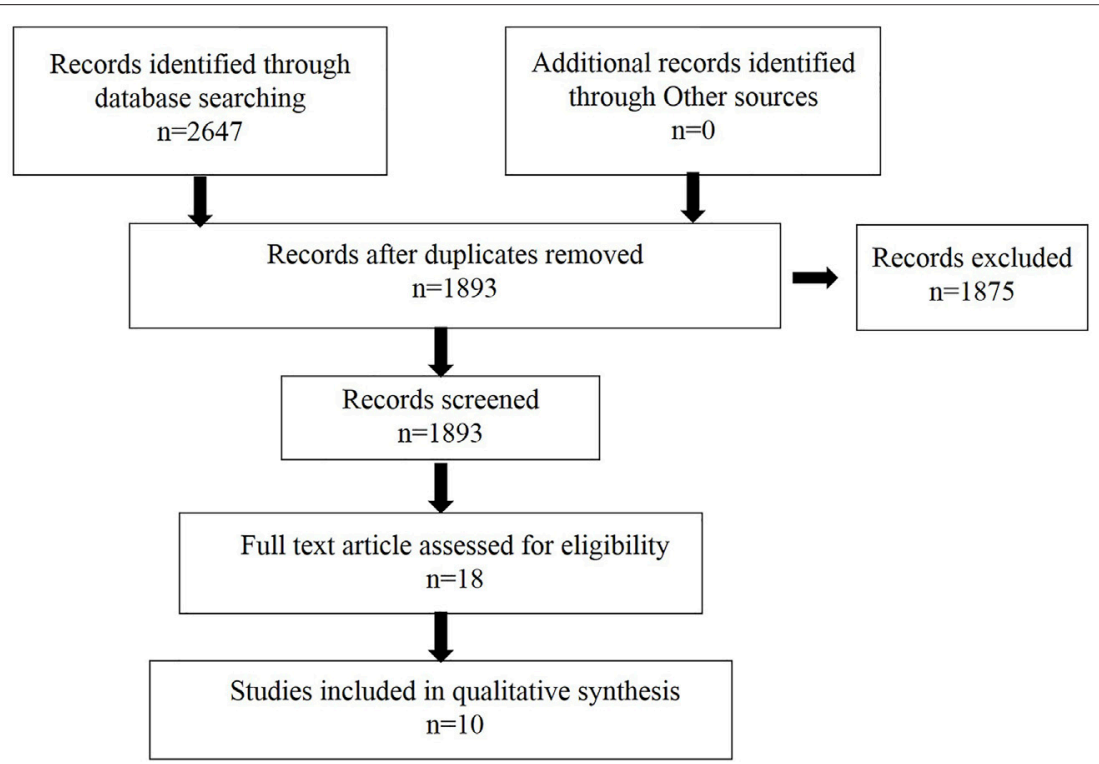

FIGURE 2 | Flow chart of literature research used to retrieve information to identify potential extracts for dementia treatment.

prohibiting mTOR pathways to protect cells exposed to oxidative stress (Preeti et al., 2017). Overexpression of sirt1 can promote nerve cell growth and regulate cellular metabolism by suppressing mTOR level (Süleyman et al., 2012). The enhanced SIRT1 activity could reduce the toxic damage imposing on nerve cells by activating AMPK-mediated autophagy process, which is essential for cell survival (Cantó et al., 2009; Dong et al., 2015). The sirt1-mediated autophagy may represent a promising therapeutic treatment to block the neurodegenerative disease (Kilic et al., 2018).

\section{TCM FOR DEMENTIA}

We searched the relevant literature to determine the extracts that may be used to treat dementia. The flow chart is shown in Figure 2, and the specific extracts are summarized in Table 1. The cholinesterase inhibitors approved by FDA have been put into clinical use for relieving the symptoms of dementia rather than therapeutic drugs (Eglit, 1999). Patients with progressive dementia may need to take more doses of cholinesterase inhibitors than before, which bring some unwanted effects, 
TABLE 1 | Herbal medicine exerts protective effect on dementia.

Herb name

Salviae Miltiorrhizae

Mulberry

Cortex Phellodendron amurensis

Ganoderma lucidum

Curcuma longa
Effective ingredients and protective mechanism

Tanshinone IIA blocking the expression of iNOS and MMP-2 protein and reduce the production of ROS (Kong et al., 2021) Resveratrol inhibit inflammation via decreasing expression of IL-1 $\beta$, IL-6, and TNF- $\alpha$ (Zhao et al., 2019)

Ethanol extract of Cortex Phellodendron amurensis promote the survival of nerve cells under toxic situation by increasing the level of Bcl-2/Bax protein (Xian et al., 2013)

Ganoderma lucidum triterpenoids attenuated neuronal damage through decreasing the expression of antioxidative protein Nrf2, HO1, and NQO1 (Yu et al., 2020)

Curcumin improved spatial learning and memory via upregulating the expression of IGF-1R, IRS-2, and Akt (Natascia et al., 2014)
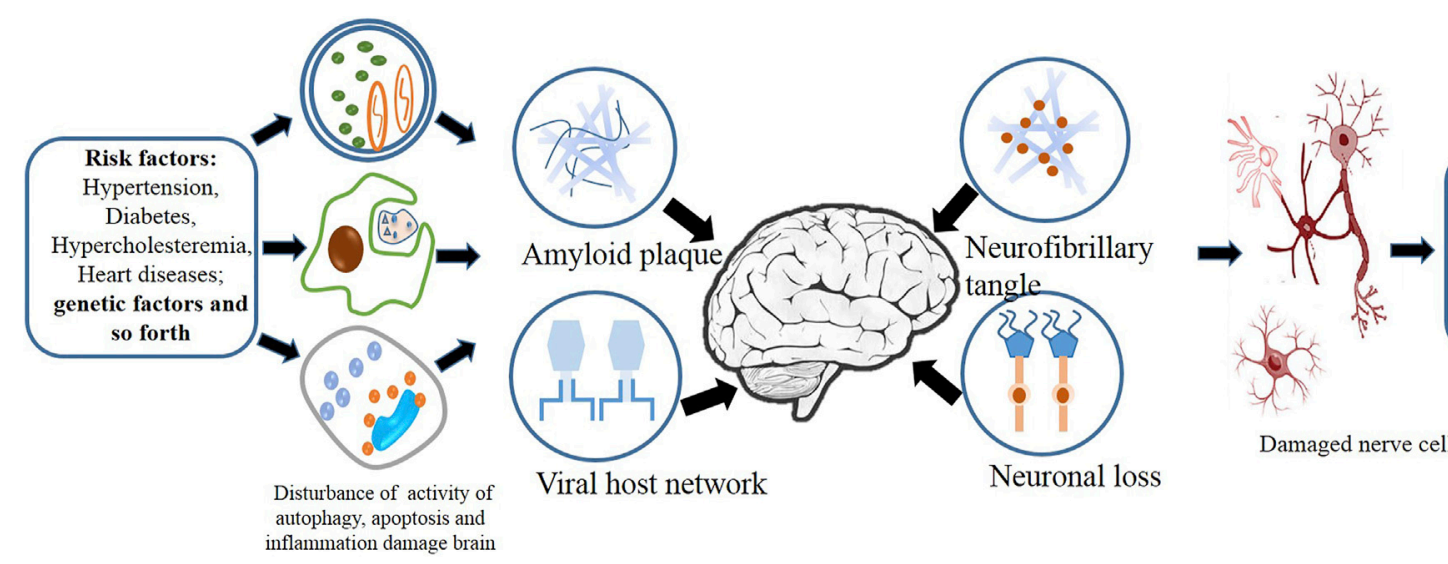

Cognitive and

memory

function impairments

Damaged nerve cells

FIGURE 3 | Factors including inflammation, autophagy, and apoptosis act on the complex cellular network of the brain.

such as nausea, vomiting, and diarrhea; these adverse events limit its clinical treatment (Skaljo et al., 2009; Lu and Herr, 2012). The combination multi-targets therapy is essential to slow down the progression of the pathogenesis of dementia from multiple perspectives (Li, 2018). TCM has become a hot point in research due to its representative of multiple targets and multiple pathway, and they play a significant role in discovering new drugs against dementia. The combination and application of various herbs are the core heart of TCM, and this process is complex, which produces different outcomes, since the complexity of formulas often reacts to different targets or pathways (Figure 3). In order to get a satisfactory efficacy, some bioactive extracts derived from herbal plants have already shown promising results in study on dementias ( $\mathrm{Ma}$ et al., 2019; Wang et al., 2020b). In this study, we will summarize some protective function of active extracts isolated form Chinese herbal plan on dementia.

\section{HM EXTRACTS ON OXIDATIVE STRESS}

Numerous studies have shown that the overgeneration of reactive oxygen species and inactive antioxidant defenses result in the failure in removing free radicals or fixing organ damage that leads to the occurrence of oxidative stress, which is considered as a key role in the pathogenesis of dementias (Mao, 2012).

Salviae Miltiorrhizae is a popular Chinese herbal plant that is widely employed in the treatment of nerve system disease including dementia (Paudel et al., 2020). Tanshinone IIA (Tan IIA) is an active compound isolated from Salviae Miltiorrhizae (Kong et al., 2021). It is reported that Tan IIA could effectively delay the progression of $\mathrm{AD}$ in rats through blocking the expression of inducible nitric oxide synthase (iNOS) and matrix metallopeptidase 2 (MMP-2) protein and also improve memory function and learning ability of rats with $\mathrm{AD}$ via reducing the generation of ROS contributed to oxidative stress (Zhi et al., 2009; Jiang et al., 2014; Tang et al., 2017).

Rehmanniae Radix is one of the commonly used Chinese herbal medicine, which could nourish kidney yin to make the brain clearer based on Chinese medicine theory (Sun et al., 2014). Rehmannioside A (ReA) is a main compound isolated from Rehmanniae Radix, which offers protection against various diseases (Jung et al., 2013). AD rats treated with Rehmannioside A have shown a great improvement in cognitive function and memory by promoting neuro growth; the mechanism is partially associated with the inhibition of NOS and superoxide dismutase (SOD) activity (Sun et al., 2014; Sun et al., 2019). 


\section{HM EXTRACTS ON APOPTOSIS}

The integrity of nerve cell is vital to the normal functional activities of the nerve system (Heydarabadi et al., 2020). Thus, progressive accumulation of impaired nerve cell will inevitably lead to a series of neurological dysfunction (Wang et al., 2019). Recent studies indicate that oxidative stress and metabolic disorder are related to the apoptosis of nerve cells, which is the main factor responsible for the neuron loss in patients with dementia. Handling apoptosis is recognized as an effective therapy in alleviating dementia (Zhang et al., 2019).

Fucoidan is a natural polysaccharide mainly isolated from brown algae with various bioactive functions, including antiinflammatory (Lee et al., 2012). A study indicated that fucoidan could protect nerve cell survival from being exposed to oxidative stress through inhibiting the expression of capase-3, which is considered to be vital role in the regulation of apoptosis (Subaraja et al., 2020).

Cortex Phellodendron amurensis (CPA), also known as "Huang Bai," is employed in Chinese medicine clinical practice as anti-heat herb for relieving inflammatory conditions and calming spirit (Xian et al., 2011). The ethanol extract of Cortex Phellodendron amurensis shows great potential in reducing neurotoxicity in beta-amyloid (A $\beta$ )-induced PC12 cells. The mechanism by which the ethanol extract of CPA affects the survival of PC12 cells are likely to be associated with increasing the level of $\mathrm{Bcl}-2 / \mathrm{Bax}$ protein and decreasing the level of capase- 3 protein so as to inhibit the occurrence of nerve cell apoptosis and exert neuroprotective effect against toxicity (Xian et al., 2013).

Salviae Miltiorrhizae-derived Tanshinone IIA (Tan IIA) exhibited a strong potential to ameliorate beta-amyloid peptides (A $\beta)$-induced cytotoxicity in PC-12 cells, which is generally used as AD research model (Dong et al., 2012). Treatment of Tan IIA in dementia is capable of protecting the brain under chronic injury and inhibiting apoptosis occurring among cells, with a repression of PI3K/Akt pathways and a downlevel of anti-autophagic regulator mTOR (Dong et al., 2012).

\section{HM EXTRACTS ON INFLAMMATION}

Inflammation observed in the development of dementia is recognized as a typical pathological feature (Lee et al., 2020). The beta-amyloid peptides (A $\beta$ )-induced inflammation in nerve cells causes the neuron loss and cognitive impairment. The injured nerve cells with low autophagy activity produce proinflammatory cytokines and chemokines, which further enhance the activity of inflammation (Lee et al., 2020). Targeted inflammation and restoration of autophagy activity could be a promising therapy in alleviating dementia.

Dioscin is an active compound derived from Polygonatum Zanlanscianense Pamp. Dioscin could remarkably prohibit the beta-amyloid peptides $(A \beta)$-induced neurotoxicity in animals with dementia and the decline in number of apoptotic cell and reactive oxygen species (ROS) production (Zhang et al., 2020). The mechanism is linked to the suppression of inflammation by downregulation of interleukin (IL)-1 $\beta$, IL-6, and tumor necrosis factor alpha (TNF- $\alpha$ ) protein. In addition, further findings indicated that the administration of Dioscin upregulates the expression of Beclin-1 and LC3-II level and sequentially restore autophagy activity (Zhang et al., 2020).

Resveratrol is a polyphenolic ascorbic acid derived from plants, such as wine, apples, and peanuts, which exert a strong protective effect against inflammation (Moussa et al., 2017). The accumulation of evidence indicated that Resveratrol is capable of blocking inflammation in the brain of animals with dementia, and the reduction in expression of IL-1 $\beta$, IL-6, and TNF- $\alpha$ (Zhao et al., 2019). In addition, resveratrol is reported to restore autophagy activity through enhancing the expression of Beclin-1 and LC3-II level and suppressing nuclear factor kappa B (NF- $\kappa \mathrm{B})$-mediated inflammation (Fisher, 2021), suggesting that restoration of autophagy activity could be used as a treatment against inflammation-induced dementia.

\section{HM EXTRACTS ON ENERGY DYSFUNCTION}

Mitochondria is a kind of organelle serving as the energy source of cellular function. Differences in the number, structure, and enzyme activity of mitochondria are existing between $\mathrm{AD}$ patients and non-AD patients (Eckert, 2019). Mitochondrial dysfunction induced by beta-amyloid peptides $(A \beta)$ has been identified as an early marker during the development of $\mathrm{AD}$, mainly characterized by the brain metabolism disorder, the dysregulation of calcium homeostasis, and the rising ROS level (Guo et al., 2019).

Curcumin is an active compound derived from Curcuma longa and shows therapeutic potential in regulating metabolism (Isik et al., 2009). Numerous studies investigating the effect of curcumin on glucose metabolism indicated that treatment of APP/PS1 double transgenic mice with curcumin enhanced glucose uptake and ameliorated impaired insulin signaling pathway in the brain and improved spatial learning and memory via upregulating the expression of IGF-1R, IRS-2, and Akt protein expression (Miyasaka et al., 2010; Natascia et al., 2014). These findings lead to a conclusion that targeting metabolism-related signaling may act as an innovative method for preventing Alzheimer's-related dementia.

Ganoderma lucidum triterpenoids (GLTs) are the major meditative compounds extracted from Ganoderma lucidum, which is documented in Chinese medicine therapy as a folk remedy based on its multifunctional health-promoting abilities (Yu et al., 2020). Multiple studies on neuroprotective effect of GLTs implied that GLTs improved cognitive impairment, attenuated nerve damage, and suppressed cell apoptosis in the nerve cells in $\mathrm{AD}$ animals through decreasing expression of anti-oxidative protein $\mathrm{Nrf} 2$, HO1, and NQO1 to inhibit the generation of ROS in the brain and promote nerve cell survival under oxidative stress situation (Yu et al., 2020). 


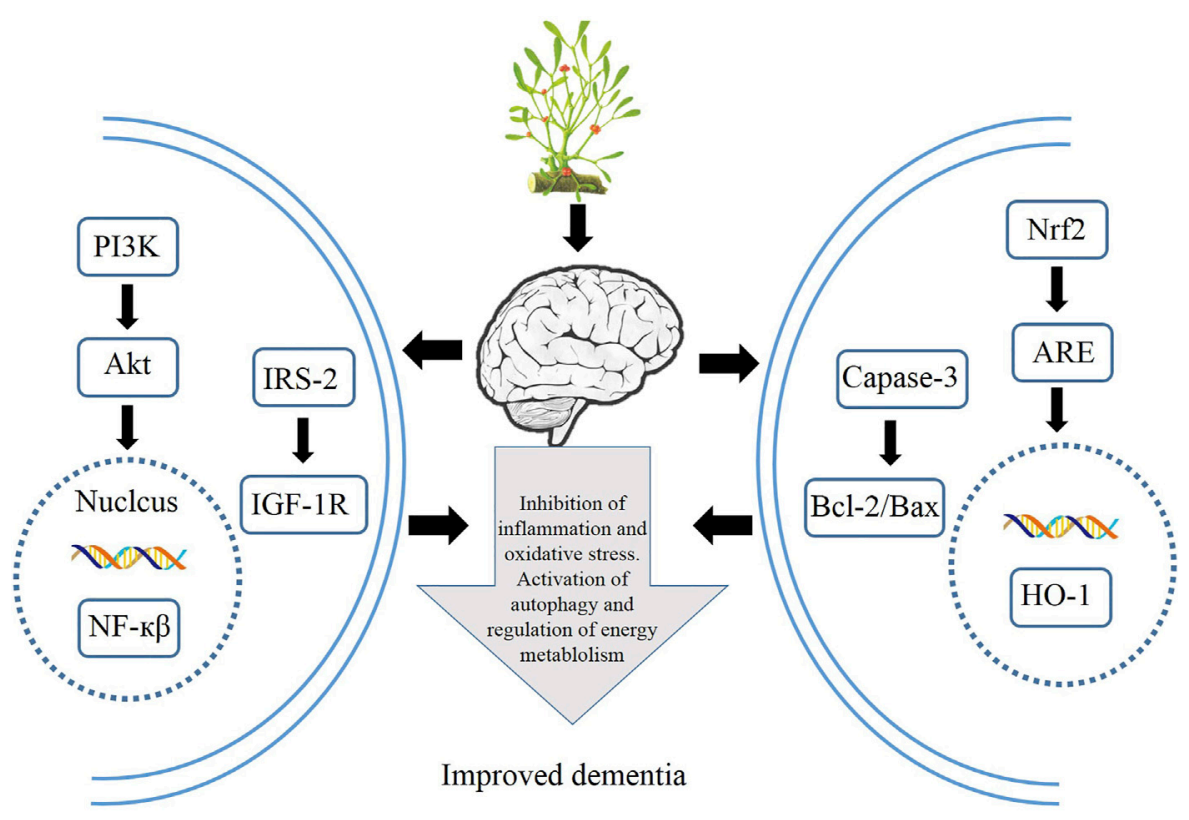

FIGURE 4 | Therapeutic applications of HMs via modulation of autophagy, inflammation, oxidative stress, and energy metabolism.

\section{CONCLUSION AND PERSPECTIVE}

HM extracts have shown its great efficacy in improving dementia, although the mechanisms are still under exploration. Recent findings highlight the role of $\mathrm{HM}$ extracts via activating various signaling pathways to attenuate cognitive barrier, spatial learning, and energy metabolism in both animal experiments and cell lines. The generally used HM extracts, such as resveratrol, Tanshinone IIA, dioscin, and curcumin, are potential candidates for new drug screening against dementia, and numerous results have demonstrated their therapeutic efficacy to attenuate nerve cell damage in both animal mode and cell lines via activating autophagy and antiinflammation and inhibiting apoptosis. The relevant mechanisms of the therapeutic application of HMs are summarized in Figure 4. Although these results sounds promising, further studies into the safety of HM extracts should be carried out, since multiple targets are involved, and most of the results are obtained from experiments; clinical practices are in great need to

\section{REFERENCES}

Alers, S., Löffler, A. S., Wesselborg, S., and Stork, B. (2012). Role of AMPK-mTORUlk1/2 in the Regulation of Autophagy: Cross Talk, Shortcuts, and Feedbacks. Mol. Cel Biol 32, 2-11. doi:10.1128/MCB.06159-11

Alirezaei, M., Kiosses, W. B., and Fox, H. S. (2008). Decreased Neuronal Autophagy in HIV Dementia: A Mechanism of Indirect Neurotoxicity. Autophagy 4, 963-966. doi:10.4161/auto.6805

Bai, X., and Zhang, M. (2021). Traditional Chinese Medicine Intervenes in Vascular Dementia: Traditional Medicine Brings New Expectations. Front. Pharmacol. 12, 689625. doi:10.3389/fphar.2021.689625 assess the therapeutic efficacy and safety on the human body. Therefore, HM extracts offer an alternative solution to the treatment of dementias via mediating various signaling pathways.

\section{AUTHOR CONTRIBUTIONS}

All authors listed have made a substantial, direct, and intellectual contribution to the work and approved it for publication.

\section{FUNDING}

This work was supported by grants from the China Postdoctoral Science Foundation (2020M681398), Youth Project of National Natural Science Foundation of China (82101602), and Medical and Health Science and Technology Development Project of Shandong Province (2018WS147).

Caberlotto, L., and Nguyen, T. P. (2014). A Systems Biology Investigation of Neurodegenerative Dementia Reveals a Pivotal Role of Autophagy. BMC Syst. Biol. 8, 65. doi:10.1186/1752-0509-8-65

Cantó, C., Gerhart-Hines, Z., Feige, J. N., Lagouge, M., Noriega, L., Milne, J. C., et al. (2009). AMPK Regulates Energy Expenditure by Modulating NAD+ Metabolism and SIRT1 Activity. Nature 458, 1056-1060. doi:10.1038/ nature 07813

Cao, J. (2010). mTOR Regulation of Autophagy. FEBS Lett. 2010, 584.

Cuervo, A. M. (2013). Autophagy and Neurodegeneration. Alzheimer $\backslash " s$ Demen. 9, P512. doi:10.1016/j.jalz.2013.04.219

Dong, H., Mao, S., Mao, S., Wei, J., Liu, B., Zhang, Z., et al. (2012). Tanshinone IIA Protects PC12 Cells from $\beta$-amyloid(25-35)-induced Apoptosis via PI3K/Akt 
Signaling Pathway. Mol. Biol. Rep. 39, 6495-6503. doi:10.1007/s11033-0121477-3

Dong, W., Wang, R., Ma, L. N., Xu, B. L., Zhang, J. S., Zhao, Z. W., et al. (2015). Autophagy Involving Age-Related Cognitive Behavior and hippocampus Injury Is Modulated by Different Caloric Intake in Mice. Int. J. Clin. Exp. Med. 8, 11843-11853.

Eckert, A. (2019). C.11.02 the Role of Mitochondria in Aging, MCI and Dementia. Eur. Neuropsychopharmacol. 29, S626-S627. doi:10.1016/j.euroneuro.2018. 11.992

Eglit, H. C. (1999). Diagnosis, Management and Treatment of Dementia.

Fisher, L. (2021). Retraction: Resveratrol Attenuates Inflammation and Reduces Matrix-Metalloprotease Expression by Inducing Autophagy via Suppressing the Wnt $/ \beta$-Catenin Signaling Pathway in IL-1 $\beta$-induced Osteoarthritis Chondrocytes. RSC Adv. 11. doi:10.1039/d1ra90015c

Guo, M., Xu, J., Wang, S., and Dong, B. (2021). Asiaticoside Reduces Autophagy and Improves Memory in a Rat Model of Dementia through mTOR Signaling Pathway Regulation. Mol. Med. Rep. 24, 284. doi:10.3892/ mmr.2021.12284

Guo, Y., Wang, S., Zhang, H., Gao, W., and Fan, F. (2019). Abstract P3016: High Glucose Induced Diabetic Dementia Is Mediated by Mitochondria Dysfunction. Hypertension 74, 3016. doi:10.1161/hyp.74.suppl_1.p3016

Han, J., Pan, X. Y., Xu, Y., Xiao, Y., An, Y., Tie, L., et al. (2012). Curcumin Induces Autophagy to Protect Vascular Endothelial Cell Survival from Oxidative Stress Damage. Autophagy 8, 812-825. doi:10.4161/auto.19471

He, C., and Klionsky, D. J. (2009). Regulation Mechanisms and Signaling Pathways of Autophagy. Annu. Rev. Genet. 43, 67-93. doi:10.1146/annurev-genet102808-114910

Heydarabadi, F. H., Abdoli, A., Gharibzadeh, S., Sayyah, M., Bashar, R., and Sheikholeslami, F. (2020). Role of Autophagy in Nerve Cell Apoptosis in Mice Infected with Street Rabies Virus. Arch. Virol. 165, 2857-2867. doi:10.1007/ s00705-020-04815-z

Isik, A. T., Celik, T., Ulusoy, G., Ongoru, O., Elibol, B., Doruk, H., et al. (2009). Curcumin Ameliorates Impaired Insulin/IGF Signalling and Memory Deficit in a Streptozotocin-Treated Rat Model. Age (Dordr) 31, 39-49. doi:10.1007/ s11357-008-9078-8

Ji, J., Tao, P., Wang, Q., Li, L., and Xu, Y. (2020). SIRT1: Mechanism and Protective in Diabetic Nephropathy. Endocr. Metab. Immune Disord. Drug Targets 21, 835. doi:10.2174/1871530320666201029143606

Jiang, P., Li, C., Xiang, Z., and Jiao, B. (2014). Tanshinone IIA Reduces the Risk of Alzheimer's Disease by Inhibiting iNOS, MMP-2 and NF-kBp65 $\mathrm{T}$-ranscription and T-ranslation in the T-emporal L-obes of $\mathrm{R}$-at M-odels of Alzheimer's D-isease. Mol. Med. Rep. 10, 689-694. doi:10. $3892 / \mathrm{mmr} .2014 .2254$

Jiang, W., and Chang, C. (2019). Discussion on Treatment of Vascular Dementia by Autophagy and Detoxification. Liaoning J. Traditional Chin. Med.

Joungmok, K., and Kun-Liang, G. (2019). mTOR as a central Hub of Nutrient Signalling and Cell Growth. Nat. Cel. Biol. 21, 63. doi:10.1038/s41556-0180205-1

Ju, J. S., and Weihl, C. C. (2010). Inclusion Body Myopathy, Paget's Disease of the Bone and Fronto-Temporal Dementia: a Disorder of Autophagy. Hum. Mol. Genet. 19, R38-R45. doi:10.1093/hmg/ddq157

Jung, E. Y., Lee, M. S., Ahn, C. J., Cho, S. H., Bae, H., and Shim, I. (2013). The Neuroprotective Effect of Gugijihwang-Tang on Trimethyltin-Induced Memory Dysfunction in the Rat. Evid. Based Complement. Alternat Med. 2013, 542081. doi:10.1155/2013/542081

Kilic, U., Elibol, B., Uysal, O., Kilic, E., Yulug, B., Sayin Sakul, A., et al. (2018). Specific Alterations in the Circulating Levels of the SIRT1, TLR4, and IL7 Proteins in Patients with Dementia. Exp. Gerontol. 111, 203-209. doi:10.1016/j. exger.2018.07.018

Kim, D., Nguyen, M. D., Dobbin, M. M., Fischer, A., Sananbenesi, F., Rodgers, J. T., et al. (2007). SIRT1 Deacetylase Protects against Neurodegeneration in Models for Alzheimer's Disease and Amyotrophic Lateral Sclerosis. EMBO J. 26, 3169-3179. doi:10.1038/sj.emboj.7601758

Kim, J., Kundu, M., Viollet, B., and Guan, K. L. (2011). AMPK and mTOR Regulate Autophagy through Direct Phosphorylation of Ulk1. Nat. Cel Biol. 13, 132. doi:10.1038/ncb2152

Kong, D., Luo, J., Shi, S., and Huang, Z. (2021). Efficacy of Tanshinone IIA and Mesenchymal Stem Cell Treatment of Learning and Memory Impairment in a
Rat Model of Vascular Dementia. J. Tradit Chin. Med. 41, 133-139. doi:10. 19852/j.cnki.jtcm.2021.01.015

Le Couteur, D. G., Doust, J., Creasey, H., and Brayne, C. (2020). Political Drive to Screen for Pre-dementia: Not Evidence Based and Ignores the Harms of Diagnosis. BMJ 347, f5125. doi:10.1136/bmj.f5125

Lee, B., Sur, B., Park, J., Shin, H., Kwon, S., Yeom, M., et al. (2012). Fucoidan Ameliorates Scopolamine-Induced Neuronal Impairment and Memory Dysfunction in Rats via Activation of Cholinergic System and Regulation of cAMP-Response Element-Binding Protein and Brain-Derived Neurotrophic Factor Expressions. J. Korean Soc. Appl. Biol. Chem. 55, 711-720. doi:10.1007/ s13765-012-2137-y

Lee, J. H., Choi, S. H., Lee, C. J., and Oh, S. S. (2020). Recovery of Dementia Syndrome Following Treatment of Brain Inflammation. Dement Geriatr. Cogn. Dis. Extra 10, 1-12. doi:10.1159/000504880

Levine, B., and Kroemer, G. (2008). Autophagy in the Pathogenesis of Disease. Cell 132, 27-42. doi:10.1016/j.cell.2007.12.018

Li, Q. (2018). Research Overview on Treating Vascular Dementia in TCM. Clin. J. Chin. Med.

Li, X. J., and Zhang, H. Y. (2009). Potential Anti-dementia Agents in Traditional Chinese Medicine. Nat. Prod. Commun. 4, 877-886. doi:10.1177/ 1934578x0900400629

Lin, S. K., Lin, P. H., Hsu, R. J., Chuang, H. C., and Liu, J. M. (2017). Traditional Chinese Medicine Therapy Reduces the Catheter Indwelling Risk in Dementia Patients with Difficult Voiding Symptoms. J. Ethnopharmacol 203, 120-126. doi:10.1016/j.jep.2017.03.040

Liu, B., Tang, J., Zhang, J., Li, S., Yuan, M., and Wang, R. (2014). Autophagy Activation Aggravates Neuronal Injury in the hippocampus of Vascular Dementia Rats. Neural Regen. Res. 9, 1288-1296. doi:10.4103/1673-5374. 137576

Lu, D. F., and Herr, K. (2012). Pain in Dementia: Recognition and Treatment. J. Gerontol. Nurs. 38, 8-13. doi:10.3928/00989134-20120113-01

Ma, J., Wang, Y., Zhang, Y., Guo, Q., Zhen, X., and Shi, M. (2019). Neuropsychological Features in post-stroke Cognitive Impairment with No Dementia Patients with Different Traditional Chinese Medicine Syndromes. J. Traditional Chin. Med. 39, 97.

Mao, P. (2012). Oxidative Stress and its Clinical Applications in Dementia. J. Neurodegener Dis. 2013, 319898. doi:10.1155/2013/319898

Miyasaka, T., Yoshimura, S., Saka, A., Shinzaki, Y., Yoshina, S., Kage-Nakadai, E., et al. (2010). Curcumin Improves Tau-Mediated Neuronal Dysfunction in Nematode. Alzheimer's Demen. 6, e27-e28. doi:10.1016/j.jalz.2010.08.086

Morita, T., Watanabe, K., Asai, M., Iwata, N., and Shirotani, K. (2010). Autophagy and its Significance on Dementia.

Moussa, C., Hebron, M., Huang, X., Ahn, J., Rissman, R. A., Aisen, P. S., et al. (2017). Resveratrol Regulates Neuro-Inflammation and Induces Adaptive Immunity in Alzheimer's Disease. J. Neuroinflammation 14, 1. doi:10.1186/ s12974-016-0779-0

Natascia, B., Simona, R., Annalisa, B., Antonella, C., Niccolò, L., Francesco, B., et al. (2014). Curcumin as a Therapeutic Agent in Dementia: A Mini Systematic Review of Human Studies. Scientific World J. 2014, 174282.

Nussenzweig, S. C., Verma, S., and Finkel, T. (2015). The Role of Autophagy in Vascular Biology. Circ. Res. 116, 480-488. doi:10.1161/CIRCRESAHA.116. 303805

Padilla, R. (2019). "Working with Elders Who Have Dementia and Alzheimer's Disease," in Occupational Therapy with Elders. Fourth Edition, 282-297. doi:10. 1016/b978-0-323-49846-3.00020-2

Paik, J. S., Ha, M., Jung, Y. H., Kim, G. H., Han, K. D., Kim, H. S., et al. (2020). Low Vision and the Risk of Dementia: a Nationwide Population-Based Cohort Study. Sci. Rep. 10, 9109. doi:10.1038/s41598-020-66002-z

Papandreou, I., Lim, A. L., Laderoute, K., and Denko, N. C. (2008). Hypoxia Signals Autophagy in Tumor Cells via AMPK Activity, Independent of HIF-1, BNIP3, and BNIP3L. Cell Death Differ 15, 1572-1581. doi:10.1038/cdd.2008.84

Paudel, P., Park, C. H., Jung, H. A., Yokozawa, T., and Choi, J. S. (2020). A Systematic Review on Anti-Alzheimer's Disease Activity of Prescription Kangen-Karyu. Drug Discov. Ther. 14, 61-66. doi:10.5582/ddt.2020.03013

Perluigi, M., Di Domenico, F., Barone, E., and Butterfield, D. A. (2021). mTOR in Alzheimer Disease and its Earlier Stages: Links to Oxidative Damage in the Progression of This Dementing Disorder. Free Radic. Biol. Med. 169, 382. doi:10.1016/j.freeradbiomed.2021.04.025 
Preeti, S., Peter, S., and Hanson, M. (2017). SIRT1 Ameliorates Oxidative Stress Induced Neural Cell Death and Is Down-Regulated in Parkinson's Disease. BMC Neurosci. 18, 46.

Qin, W., Yang, T., Ho, L., Zhao, Z., Wang, J., Chen, L., et al. (2006). Neuronal SIRT1 Activation as a Novel Mechanism Underlying the Prevention of Alzheimer Disease Amyloid Neuropathology by Calorie Restriction. J. Biol. Chem. 281, 21745-21754. doi:10.1074/jbc.M602909200

Romeo, M. A., Faggioni, A., and Cirone, M. (2019). Could Autophagy Dysregulation Link Neurotropic Viruses to Alzheimer's Disease? Neural Regen. Res. 14, 1503-1506. doi:10.4103/1673-5374.253508

Sahyouni, R., Chen, J., Verma, A., and Brown, N. (2021). Alzheimer's Disease Decoded: The History, Present, and Future of Alzheimer's Disease and Dementia, Alzheimer's Disease Decoded: The History, Present, and Future of Alzheimer's Disease and Dementia.

Salminen, A., and Kaarniranta, K. (2009). SIRT1: Regulation of Longevity via Autophagy. Cell Signal 21, 1356-1360. doi:10.1016/j.cellsig.2009.02.014

Shen, Y. J., and Li, C. Y. (2005). Experimental Study of Traditional Chinese Medicine in the Treatment of Vascular Dementia. Zhongguo Zhong Yao Za Zhi 30, 725-728.

Skaljo, E., Hodzic, M., and Bektas, I. (2009). “Treatment Guidelines for Agitation in Dementia Patients," in International Conference on Ultra Modern Telecommunications \& Workshops.

Subaraja, M., Anantha Krishnan, D., Edwin Hillary, V., William Raja, T. R., Mathew, P., Ravikumar, S., et al. (2020). Fucoidan Serves a Neuroprotective Effect in an Alzheimer's Disease Model. Front. Biosci. Elite Ed. 12, 1-34.

Süleyman, C., Hai, E., Dang, W., Le, S., and Widlund, A. L. (2012). The Relation between Resveratrol, Sirt1, mTOR and Autophagy.

Sun, M., Shen, X., and Ma, Y. (2019). Rehmannioside A Attenuates Cognitive Deficits in Rats with Vascular Dementia (VD) through Suppressing Oxidative Stress, Inflammation and Apoptosis. Biomed. Pharmacother. 120, 109492. doi:10.1016/j.biopha.2019.109492

Sun, W., Hongmei, A. N., and Hospital, L. (2014). Effects of Prepared Radix Rehmanniae and Related Herbal Formula in Treatment of Senile Dementia. Chin. Arch. Traditional Chin. Med..

Tang, L. M., Wang, L. X., Wang, Z. Y., Sun, L. F., Pan, X. D., and Pan, G. Q. (2017). Tanshinone IIA Ameliorates lead (Pb)-Induced Cognitive Deficits and Oxidative Stress in a Rat Pup Model. Bratisl Lek Listy 118, 196-201. doi:10. 4149/BLL_2017_039

Wang, H., Yu, H., Song, K., Xiong, F., and Zhang, H. (2020). Traditional Chinese Medicine for Mild Cognitive Impairment: A Protocol for Systematic Review and Network Meta-Analysis. Medicine (Baltimore) 99, e22187. doi:10.1097/ MD.0000000000022187

Wang, Q. C., Lu, L., and Zhou, H. J. (2019). Relationship between the MAPK/ERK Pathway and Neurocyte Apoptosis after Cerebral Infarction in Rats. Eur. Rev. Med. Pharmacol. Sci. 23, 5374. doi:10.26355/eurrev_201906_18206

Wang, W., Qiao, O., Ji, H., Zhang, X., Han, X., Zhang, Y., et al. (2021). Autophagy in Vascular Dementia and Natural Products with Autophagy Regulating Activity. Pharmacol. Res. 170, 105756. doi:10.1016/j.phrs.2021.105756

Wang, X. X., Zhang, B., Xia, R., and Jia, Q. Y. (2020). Inflammation, Apoptosis and Autophagy as Critical Players in Vascular Dementia. Eur. Rev. Med. Pharmacol. Sci. 24, 9601-9614. doi:10.26355/eurrev_202009_23048
Xian, Y. F., Lin, Z. X., Ip, S. P., Su, Z. R., Chen, J. N., and Lai, X. P. (2013). Comparison the Neuropreotective Effect of Cortex Phellodendri Chinensis and Cortex Phellodendri Amurensis against Beta-Amyloid-Induced Neurotoxicity in PC12 Cells. Phytomedicine 20, 187-193. doi:10.1016/j.phymed.2012.09.028

Xian, Y. F., Mao, Q. Q., Ip, S. P., Lin, Z. X., and Che, C. T. (2011). Comparison on the Anti-inflammatory Effect of Cortex Phellodendri Chinensis and Cortex Phellodendri Amurensis in 12-O-Tetradecanoyl-Phorbol-13-Acetate-Induced Ear Edema in Mice. J. Ethnopharmacol 137, 1425-1430. doi:10.1016/j.jep.2011. 08.014

Xu, Y., Wang, Q., Qu, Z., Yang, J., Zhang, X., and Zhao, Y. (2019). Protective Effect of Hyperbaric Oxygen Therapy on Cognitive Function in Patients with Vascular Dementia. Cel Transpl. 28, 1071-1075. doi:10.1177/ 0963689719853540

Yu, N., Huang, Y., Jiang, Y., Zou, L., Liu, X., Liu, S., et al. (2020). Ganoderma Lucidum Triterpenoids (GLTs) Reduce Neuronal Apoptosis via Inhibition of ROCK Signal Pathway in APP/PS1 Transgenic Alzheimer's Disease Mice. Oxid Med. Cel Longev 2020, 9894037. doi:10.1155/2020/9894037

Zhang, H., Zhang, Y., Zhou, L., Ruipeng, W. U., Wei, J., Juanjuan, L. U., et al. (2019). Effects of Total Alkaloids of Rhizoma Corydalis on Hippocampal Cell Apoptosis and Cognitive Function in Vascular Dementia Rats. J. Zhengzhou Univ. (Medical Sciences).

Zhang, Z., Han, K., Wang, C., Sun, C., and Jia, N. (2020). Dioscin Protects against A $31-42$ Oligomers-Induced Neurotoxicity via the Function of SIRT3 and Autophagy. Chem. Pharm. Bull. (Tokyo) 68, 717-725. doi:10.1248/cpb.c2000046

Zhao, Z., Zou, Z., Mao, C., Liu, J., Hao, G., Shi, Z., et al. (2019). Resveratrol Alleviates the Nervous System Inflammation by SIRT1 in Rats with Subarachnoid Hemorrhage. Chin. J. Neuroanat.

Zhi, H., Pan, Z., and Lu, W. (2009). Neuroprotective Effects of Tanshinone IIA on Vascular Dementia in Rats. Pharmacol. Clin. Chin. Materia Med.

Zhu, A. H., Tian, J. Z., Zhong, J., Yang, C. Z., Shi, J., and Yin, J. X. (2006). A Clinical Study on a Randomized, Double-Blind Control of Chinese Medicine Granules in Treatment of Vascular Dementia. Zhongguo Zhong Yao Za Zhi 31, 1722.

Conflict of Interest: The authors declare that the research was conducted in the absence of any commercial or financial relationships that could be construed as a potential conflict of interest.

Publisher's Note: All claims expressed in this article are solely those of the authors and do not necessarily represent those of their affiliated organizations, or those of the publisher, the editors, and the reviewers. Any product that may be evaluated in this article, or claim that may be made by its manufacturer, is not guaranteed or endorsed by the publisher.

Copyright (c) $2022 \mathrm{Tao}, \mathrm{Ji}, \mathrm{Gu}$, Wang and Xu. This is an open-access article distributed under the terms of the Creative Commons Attribution License (CC $B Y)$. The use, distribution or reproduction in other forums is permitted, provided the original author(s) and the copyright owner(s) are credited and that the original publication in this journal is cited, in accordance with accepted academic practice. No use, distribution or reproduction is permitted which does not comply with these terms. 